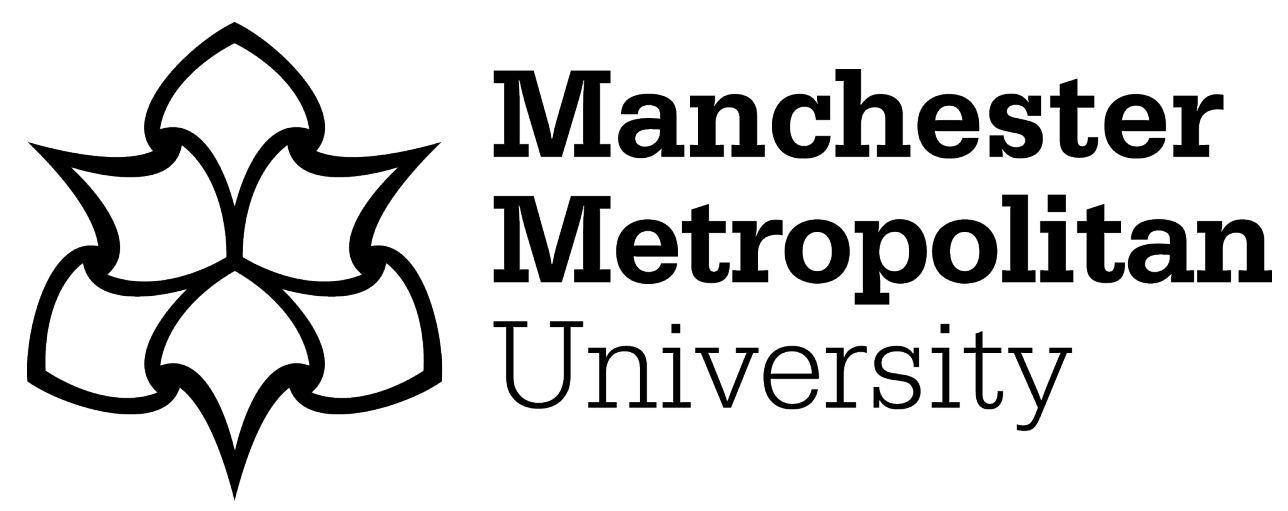

Melthis, John, Tang, Stephen, Yang, Po, Hanneghan, Martin and Carter, Chris (2016) Topologies for combining the internet of things and serious games. Journal of Intelligent \& Fuzzy Systems, 31 (5). pp. 2685-2696. ISSN 1064-1246

Downloaded from: https://e-space.mmu.ac.uk/623243/

Version: Accepted Version

Publisher: IOS Press

DOI: https://doi.org/10.3233/jifs-169108

Please cite the published version 


\title{
Topologies for combining the Internet of Things and Serious Games
}

\author{
John Melthis ${ }^{1}$, Stephen Tang ${ }^{2}$, Po Yang ${ }^{3}$, Martin Hanneghan ${ }^{4}$ and Chris Carter ${ }^{5}$ \\ Department of Computer Science, \\ Faculty of Technology and Environment, \\ Liverpool John Moores University, \\ James Parsons Building, Byrom Street, Liverpool, L3 3AF, UK.
}

Email: j.melthis@2015.ljmu.ac.uk ${ }^{1}$, o.t.tang@ljmu.ac.uk ${ }^{2}$, p.yang@1jmu.ac.uk ${ }^{3}$, m.b.hanneghan@ljmu.ac.uk ${ }^{4}$, c.j.carter@ljmu.ac.uk ${ }^{5}$

\begin{abstract}
Serious Games have been established over recent years as a means of utilising gaming for applications other than entertainment. With the emergence of the Internet of Things (IoT) paradigm, a new direction for serious games arises, where data gathered from the physical environment can be utilised towards new novel applications.

This literature survey uncovers existing topologies that can be applied for combining IoT with Serious Games. This paper presents findings from extensive research into IoT, Serious Games, Pervasive Games and Gamification, IoT topologies and Wireless Sensor Networks (WSN), to identify the requirements of a topology for Serious Games and IoT. By understanding the topological requirements for combining IoT and Serious Games, the development process is reduced, allowing for the advancement in the mentioned field.

Three topologies are presented for combining IoT with Serious Games and a detailed topology for developing a Serious Game that monitors student attendance is presented. Also included, is an insight into the new paradigm of Smart Serious Games (SSGs). This paper will aid future research and development in SSGs determine effective network topologies.
\end{abstract}

Index Terms-IoT; Serious Games; Topology; Smart Serious Games;

\section{INTRODUCTION}

IoT promises a future of interconnectivity between heterogeneous devices and data services. This interconnectivity will allow for better analysis of data driven applications and prospects new software solutions that could not have been achieved without IoT. IoT consists of interconnected devices or Things that operate in Smart Environments and communicate data with virtual identification and/or personalities [1]. In addition, IoT accounts for an ecosystem which is comprised of middle-ware [2], users and interconnected devices.

Serious Games have been defined as computer games built for non-entertainment domains, and have a presence in industries including health, advertisement, training, education, science, research, and others [3]. By harnessing the power of entertainment that gaming provides, serious games and gamification have provided a number of research and industrial solutions [4], [5], [6], [7].

In a truly interconnected IoT environment, Serious Games could harvest and analyse data from players physical worlds and present it so behaviour can be positively manipulated.
The combination of Serious Games and IoT has recently been termed as Smart Serious Games (SSGs) [8]. SSGs have been defined as the merger of smart technologies, including devices and services, and the principles of Serious Games [8]. This literature details the combination of the advantages of both technologies and its future utilisations including; analytics for cooperations, a tool for solving serious problems and others.

As this research area is new, literature on SSGs is limited, with some research project beginning to include the term as future works [9], [10]. It is very important to continue research into the combination of SSGs as IoT brings better data acquisition and a pervasive experience, elements that create quantitative results with less intrusive methods for obtaining them.

To develop a SSG, research into IoT, data analytics and Serious Games is required. An area of research that must be addressed are topologies for developing a Serious Game combined with IoT. Research into IoT topologies [?] and game topologies [11] exist, however none has been conducted on appropriate topologies for SSGs, nor have current topologies been evaluated for this purpose. This paper focuses on this area; specifically topologies for SSGs, and surveys existing topologies that can be utilised, based on the latest research. By determining the appropriate topologies for combining Serious Games and IoT or SSGs, a foundation for future development in the research field can be achieved, as it is vital to consider the network topology of any research principle that merges with the IoT paradigm.

This paper follows the following outline. Section II reviews literature on IoT and Serious Games. Section III presents current topologies, utilised in a variety of applications and analyses the effectiveness of incorporating these topologies for SSGs. Topologies have been sourced from networking games, WSNs and Human - centric WSNs. Requirements for SSG topologies are outlined in Section IV. Topologies for SSGs are presented in Section V, outlining three different types of topologies that can be used for combining Serious Games and IoT. Section VI discusses a potential SSG application and illustrates its topology. The conclusive remarks are made in Section VII. 


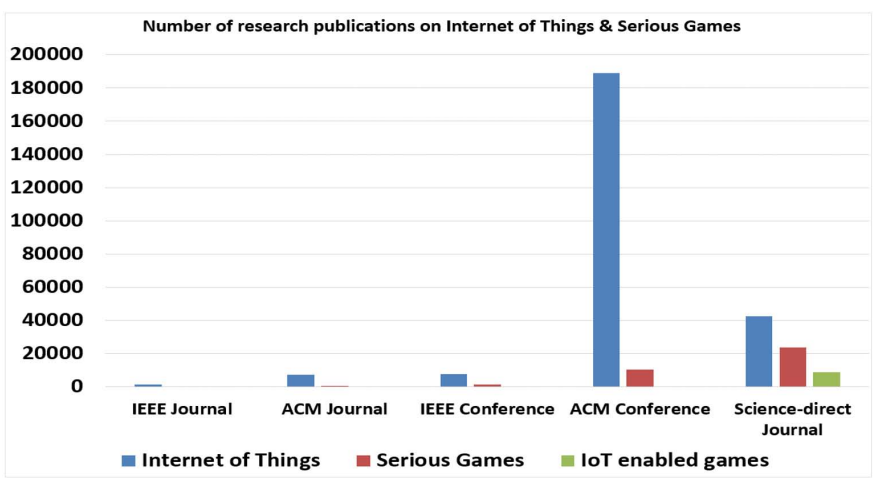

Fig. 1. Number of Journal and Conference articles related to IoT and Serious Games from 2000 to 2015 (IoT-Internet of Things, Serious Games, IoT enabled Games)

\section{REVIEW OF INTERNET OF THINGS AND SERIOUS GAMES}

This paper aims to provide a systematic review on current literature of PARM from an IoT layer-based perspective, as shown in Fig.1. We undertook an extensive literature review by examining relevant articles from major academic databases. (IEEE Xplore, ACM, Springer digital library and ScienceDirect). Key search terms include the key words Internet of Things, Serious Games, IoT Game and a wide range of other technologies. The results are shown in Fig.1, which indicate that both topics of Internet of Things and Serious Games are widely popular in literature, but there are few studies on investigating the combination of them. It is worth exploring potential research issues in this area.

\section{A. Internet of Things}

The term Internet of Things (IoT) was firstly introduced by Kevin Aston [12] in 1999, aiming at identify unique objects and their virtual representations in an internet-like structure. Radio-frequency identification (RFID) technology [13], [14], [15], [16], [17] was firstly proposed to label these objects for tracking their location [13] [14], movements [17], or even ecosystem experience [14].

Recently, through the development of sensing technology, more things are able to be connected and labelled on the internet. The scope of IoT has been extended to: people-tothings, things-to-things, machine-to-machine amongst others. To elaborate, IoT enables the connectivity of anything from any time to any place. Nowadays, researchers have expanded IoT with more technologies including sensors, network, data analysis, and various applications. Its applications include diverse and wide fields such as: industries [18], environments [19], cities [20], [21], transportation [22] and healthcare [23], [24], [25].

We normally categorise IoT enabled technologies, in a fourlayered IoT architecture [1] with a sensing layer, networking layer, data processing layer, and application layer. Each layer of this IoT architecture is concerned with a wide board of technologies as itemised below:

- Sensing layer: Integrated with existing sensory devices (RFID, actuators, wearable devices, mobile phone, bar- code and others) to sense and collect the physical data from the real world [26].

- Networking layer: Provides basic networking and data communication over a wireless or wired network, including WWAN, WPAN, WLAN [27].

- Data processing layer: Concerns a number of processing steps for handling raw sensory data [28], including the construction of data storage centres, search engines, smart decisions and data mining approaches.

- Application layer: Provides an interface for user interaction [29], [30].

While IoT enabled technologies cover a variety of fields and areas, this paper focuses on the combination with serious games, mainly relating to the networking and application layer.

\section{B. IoT and Serious Games}

By combing IoT into Games, a seamless exchange of information between virtual services, real items and products is established [31]. This idea has been studied for couple of years, by using a variety of wearable devices or sensors to implement an augmented/virtual reality of gaming experience.

Modern computer games of all genres tend to utilise game engines for their development. Game engine, is the term applied to an extensible software library which promotes productivity for game developers, allowing them to reuse proven and tested software libraries to produce a variety of computer games within the scope of a software framework [32] [33].

There are a number of game engines currently available of which a small sample includes the Unreal Engine (commonly used for its extensive tools and features), DICEs Frostbite Engine (widely utilised for Electronic Arts games such as Battlefield), Microsofts XNA Game Studio (utilised for the development of Xbox and PC games through C\# and Visual Studio) and Unity (primarily known for its ease of use and cross-platform game deployment) [32]. A game engine could be essential to develop and deploy a Serious Game that connects with IoT, dependant on the assets and virtual environment.

Serious games refers to the use of game techniques or games technology for non-entertainment purposes. Applications include games for health, advertisement, training, education, science, research, production and work, in which games technologies are used specifically for improving accessibility of simulations, modelling environments, visualisation, interfaces, delivery of messages, learning and training, and productive activities such as authoring, development or production [34]. Notable examples of serious games applications include Foldit, a protein folding game that crowd-sources gamers to help solve real-life problems [35], Supercharged!, which is designed to teach physics to middle school children [36] and Virtually Betters virtual reality exposure therapy, which uses virtual reality and games technology to help treat war veterans who suffers from Post-Traumatic Stress Disorder [37].

Serious Games may be developed into pervasive games to include IoT. Computer games, played through converging 
physical and virtual worlds by utilising receptive technology can be defined as pervasive games [38]. Commonly, pervasive games utilise Augmented Reality (AR), to bridge virtual and physical worlds and increase user impressiveness [39]. Serious games for healthcare utilise wireless networks to aid in rehabilitation and promote fitness, amongst other research topics. Body area networks (BAN) are utilised for medical, lifestyle and entertainment purposes. BANs comprise of sensors and actuators, placed in, on and around the body [40].

As an alternative to Serious Games, Gamification is the process of improving services and/or products with the use of game elements for enhanced user immersion and behaviour [41]. The research to be conducted can aid in the combination of pervasive games and IoT and Gamification and IoT as Serious Games shares attributes with these types of games.

Recent advances in IoT offer the potential for service specific applications of serious games. For instance, the utilisation of Barcode technology into an online game [42]. R. Adelmann et.al. developed a social network game (Product Empire) which motivates users to scan barcodes and to enter basic product information. The experiment results shown that this attempt of integrating IoT into Serious Game generates a potentially open product repository, further improving customer shopping experiences.

\section{BACKGROUND AND CURRENT TOPOLOGIES}

IoT can help serious games become more pervasive, and extend gaming from the virtual, to the physical world. Computer games, played through converging physical and virtual worlds through the use of receptive technology can be defined as pervasive games [43]. By making Serious Games more pervasive, players' input are captures in a less intrusive manner, allowing players to focus on gameplay and therefore improving player immersion [39].

IoT, however, can also improve gamification. This means that a pervasive, gamified real world task can be developed to positively manipulate player behaviour.

Manipulating player behaviour can be achieved in a learning environment. A survey into serious game for education by $\mathrm{C}$. Girald et.al. highlighted an issue of obtaining quantitative data in Serious Games research in order to generate meaningful results. Only $27 \%$ of studies shown an improvement in learning [6]. By incorporating IoT with Serious Games, interconnected sensors act as a source of quantitative data, providing that appropriate data analysis is undertaken.

To develop an effective SSG solution, available topologies must be researched and their appropriateness for service analysed. This section surveys existing topologies that can be utilised or adapted to accommodate for SSGs.

\section{A. Client Server and Peer-to-Peer Topologies}

The online gaming client - server architecture included in literature by Armitage G. et. al, provides an example of existing topologies, where principles can be applied for a SSG. In client - server architectures, clients require a service from the server. Clients cannot communicate directly, but rather send messages through the server [11]. A large limitation of the client server architecture is its focus on the server. If the server was to become unresponsive and disconnect, the whole network would become redundant until the server can either be repaired or replaced.

The adoption of peer-to-peer architectures for SSGs is another topology for consideration. A peer-to-peer topology for Massively Multiplayer Online Games suggested by Yahyavi A. and Kemme B., offers greater scalability over client-server architectures, due to a better distributed network load [44]. IoT is expanding rapidly, with continuous developments in low energy sensor devices and data analytics. To accommodate for the hardware included in the future of IoT ecosystem, scalability should be considered as a key element. This favours the use of elements from a peer-to-peer topology that harness scalability for a SSG.

The MOPAR project developed a hybrid architecture for online games that focuses on interest management, utilising peer-to-peer mobile technologies [45]. The aforementioned project did not produce a topology but resulted in a hybrid solution with key advantages in scalability and fault tolerance over previous approaches, values that prove beneficial for a SSG game that utilises mobile technologies.

\section{B. Cloud Topologies}

The research of D. Misha et.al. into the utilisation of cloud architecture for online games, suggested a two-tier cloud architecture where Tier 1 is formed by public cloud services and Tier 2 consists of the servers that are closest to any given gamer [46]. The paper of D. Misha et.al. did identify limitations in their proposition related to prising and potential security risks stemming from the cloud. A full cloud integration may prove beneficial for SSGs due to the scalability and decentralised nature of such an topology. Current cost restrictions favour a hybrid architecture, particularly for research purposes.

Cloud-centric IoT presents the idea of cloud computing forming the core of IoT with users, sensor networks, middleware and private clouds completing the paradigm [30]. The framework J.Gubbi et.al. developed creates a scalable IoT for multiple applications. When considering IoT from a scaled back perspective, such a representation becomes accurate. For SSGs consideration must be given to smaller infrastructure also, as not all even $t$ driven games that require Io $T$ may require the cloud. For instance, a serious game may operate with a localised server, using sensors and actuators placed in a single room and open source data which fundamentally affect gameplay.

\section{Wireless Sensor Network Topologies}

When utilising WSN in an IoT solution, the level of integration required must be determined. C. Alcaraz et. al. have outlined Two approaches for integrating IoT and WSN: Stack-Based and Topology-Based [47].

Focusing on topology based approaches, two solutions were outline: Hybrid and Access Point. Hybrid solutions 
TABLE I

TOPOLOGY COMPARISON

\begin{tabular}{|c|c|c|c|c|c|c|c|c|}
\hline \multicolumn{9}{|c|}{ TOPOLOGY COMPARISON } \\
\hline & \multirow{2}{*}{$\begin{array}{l}\text { SURVEYED } \\
\text { TOPOLOGIES }\end{array}$} & \multicolumn{7}{|c|}{ Topology } \\
\hline & & $(7)$ & $(8)$ & (9) & $(10)$ & (11) & $(12)$ & $(13)$ \\
\hline \multirow{7}{*}{ 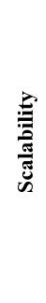 } & sensor compatibility & & & & $\checkmark$ & $\checkmark$ & $\checkmark$ & $\checkmark$ \\
\hline & web datasets & & & & $\checkmark$ & $\checkmark$ & & \\
\hline & energy efficiency & & & & & $\checkmark$ & $\checkmark$ & $\checkmark$ \\
\hline & node flexibility & & & & $\checkmark$ & $\checkmark$ & $\checkmark$ & $\checkmark$ \\
\hline & peak demand & $\checkmark$ & $\checkmark$ & & $\checkmark$ & & & \\
\hline & peer bandwidth & $\checkmark$ & $\checkmark$ & $\checkmark$ & & & & \\
\hline & mobility & & & & $\checkmark$ & $\checkmark$ & & $\checkmark$ \\
\hline \multirow{4}{*}{ \&ै } & $\begin{array}{l}\text { low power } \\
\text { consumption }\end{array}$ & & & & $\checkmark$ & $\checkmark$ & $\checkmark$ & $\checkmark$ \\
\hline & resource optimisation & $\checkmark$ & & $\checkmark$ & $\checkmark$ & $\checkmark$ & $\checkmark$ & $\checkmark$ \\
\hline & parameter based QoS & $\checkmark$ & $\checkmark$ & $\checkmark$ & $\checkmark$ & & & \\
\hline & priority based QoS & $\checkmark$ & $\checkmark$ & $\checkmark$ & $\checkmark$ & & & \\
\hline \multirow{3}{*}{ 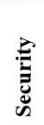 } & low power protocols & & & & & $\checkmark$ & & \\
\hline & encryption & $\checkmark$ & & & & $\checkmark$ & & \\
\hline & physical placement & $\checkmark$ & $\checkmark$ & $\checkmark$ & & & & \\
\hline
\end{tabular}

feature networked nodes that can connect to the Internet autonomously, providing the network with redundancy and intelligence. Access Point Solutions rely on a sink node for connectivity to the Internet, meaning nodes at the edge of the network have less capabilities and specify on the data attempted to be captured, for example: movement [47]. The research of C. Alcaraz et. al. confirms that the potential of WSN is unleashed when integrated into IoT.

Developing a large scale WSN is currently challenging due to the variety in domains, vendors and standard [2]. From the Serious Game context, an interconnected WSN creates new possibilities for gamifying new scenarios and creating pervasive games that interact with the physical and virtual environment.

An effective WSN topology should be scalable, energy efficient and reliable [1]. D. Zhang et. al. introduced a new approach for a weighted network topology in WSN which identified sink holes amongst sensors, and defined a new edge weight [48]. By utilising the results generated, energy holes can be avoided in IoT networks. The scale of a SSG network would form the likelihood of energy holes. For further research a localised hybrid topology will be utilised, detailed in the following section. Larger SSG networks that gather a greater amount of real-world data should utilise a weightedIoT topology.

Consideration must be given to Human-centric Wireless Sensor Networks (HWSN) when considering a topology for SSGs. Unlike WSNs, HWSNs require that every networked node sends information to a Human Based Sensor (HBS) [49]. This type of topology would be ideal for games that monitor specific body parts or require body scanning, such as brain activity. HWSN topologies can benefit games that require players' current location.

\section{REQUiREMENTS FOR SMART SERIOUS GAMES TOPOLOGIES}

A topology for games must consider data packet loss, latency and jitter to create a fluid gaming experience [11]. The requirements for SSG topologies have not yet been defined in current literature. We propose the following key requirements for an SSG topology, based on the conducted research:

1) Scalability: Scalability is an ambiguous concept, as it is service specific. For a scalable SSG topology, scalability must consider WSN and gaming network attributes. To incorporate IoT into a game data must be sourced through sensors, either directly or indirectly. By indirect sourcing of data we consider open datasets available on the Internet. To account for the network demands of a sensor based solution, WSN topology requirements are essential.

A scalable WSN topology allows nodes to be added without major reconstruction or development [1] by accounting for the node density of a given topology [50]. Furthermore consideration is given to the energy efficiency of an WSN topology. To achieve efficiency, the least amount of power should be utilised for the network to operate reliably, and sleep modes should be operational where possible for nodes that are not constantly active [1].

Aside from the sensory requirements of a SSG topology, the gaming network demands must be met. From a gaming perspective, a scalable network must adapt to peak demand and peer bandwidth [51]. Depending on the size of a player community, demand can sore during off-school hours and significantly affect gameplay. Peer bandwidth will affect data demanding games that require large files to be sent back and forth from a server. Contrary to a traditional peer-to-peer network, a SSG topology must consider mobility in nodes [52] as household devices adopt more sensory equipment.

2) Quality of Service $(Q o S)$ : SSG topologies must adhere to Quality of Service (QoS). The required components to achieve QoS for SSGs can be sourced by considering WSNs and networks for online games.

From a WSN perspective, QoS can be achieved by considering the low power consumption attribute of sensor networks [50]. Research by D. Chrstin' et.al, recommends heterogeneous sensors contribute to assure QoS by optimising network resources [53]. When considering the gaming element of a SSG topology, a lack of QoS can detrimentally reduce the effectiveness of an application, for example, latency on realtime, real-world data required to effect gameplay. Networks for online games implement parameter-based and prioritybased QoS [11], to ensure a reduction in latency and provide a fluid gaming experience.

By incorporating the aforementioned elements of WSN and online game networks for QoS, a blend that is sufficient for accommodating future SSG applications that support multiplayer and data from sensor and mobile devices is generated.

3) Security: The security of a topology can be divided into two categories; physical and network. The physical placement of sensors and actuators must consider vandalism and burglary, particularly when expensive hardware is involved. Security of 
smart devices must also be considered as they become embedded into SSG solutions. Major manufacturers offer services such as remote wiping to prevent unauthorised access, however SSG solutions should offer players the ability to remove their own devices from a network to maximise security. Allowing users to manipulate their own smart devices on a network can also improve scalability.

From a networking perspective, sensors and actuators with Internet connectivity must utilise protocols and encryption mechanisms that prevent malicious attacks and theft of personal data. Due to the low power nature of sensors and actuators, research has underline the necessity of new security mechanisms that can operate at low power [53]. Further security requirements will be service specific.

\section{IOT TOPOlOGies for Serious Games}

The reviewed topologies and requirements in previews sections highlight the incompatibility of traditional topologies with the requirements of IoT topologies for Serious Games. The following section presents updated variations of notable topologies for SSGs.

1) Client-Server and Peer-to-peer: In Section "Current Topologies" client-server and peer-to-peer topologies for online games have been reviewed. Figure 2 proposes a client server architecture and a peer-to-peer client - server hybrid topology that is adapted for the IoT ecosystem. The proposed architecture will share elements of a peer-to-peer architecture but will fundamentally differ in the roles clients have within the network. Clients will not be formed solely by computers but rather by a combination of sensors and computational devices. An additional difference is the intercommunication between clients. Clients will need to communicate with each other directly.

2) Interconnected Access Point: For SSGs, three different topologies can be considered. The interconnected access point topology, illustrated in Fig. 3, is better utilised for solutions that involve multiple users and locations such as an augmented reality, Massive Multi-player Online (MMO) game. This is due to the small clusters of sensors and actuators formed with the sink node, allowing a number of low powered nodes to be placed in a variety of locations.

Father.io ${ }^{1}$ examples an augmented reality MMO game that could utilise an interconnected access point topology. Focused on merging smart devices with Personal Computer (PC) gaming, Father.io utilises smartphones to augment players physical perspective and enable them to engage in an MMO shooter. The video trailer showcases a smartwatch utilised ingame alongside a smartphone. To achieve this data must flow between smart devices to allow for a fluid gaming experience. Data could be synced via a sink node, or the smartphone could be utilised as such, however devices could also be connected autonomously, as illustrated in the hybrid topology. Father.io is currently a concept but presents an insight into the future of IoT games.

\footnotetext{
${ }^{1}$ https://www.indiegogo.com/projects/father-io-massive-multiplayer-lasertag/, accessed 30/03/2016
}

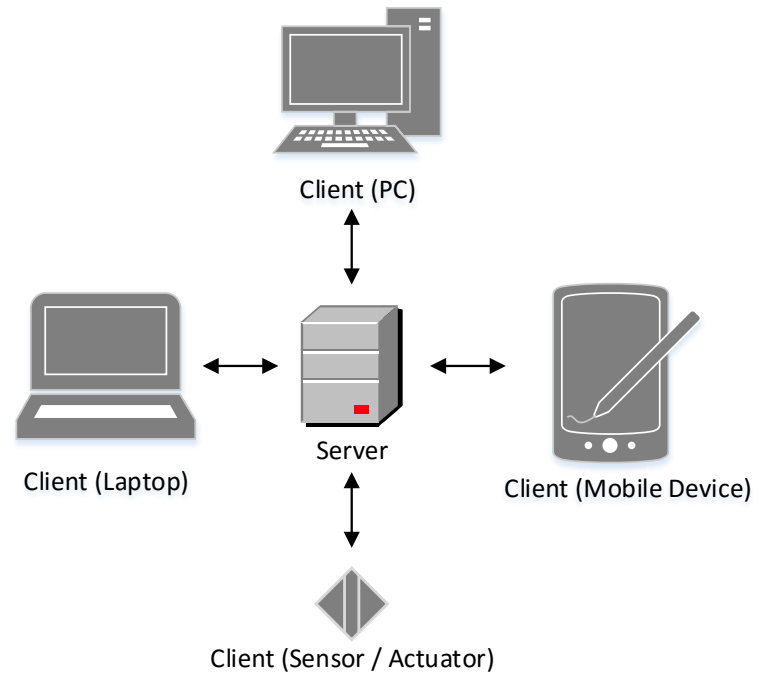

(a)

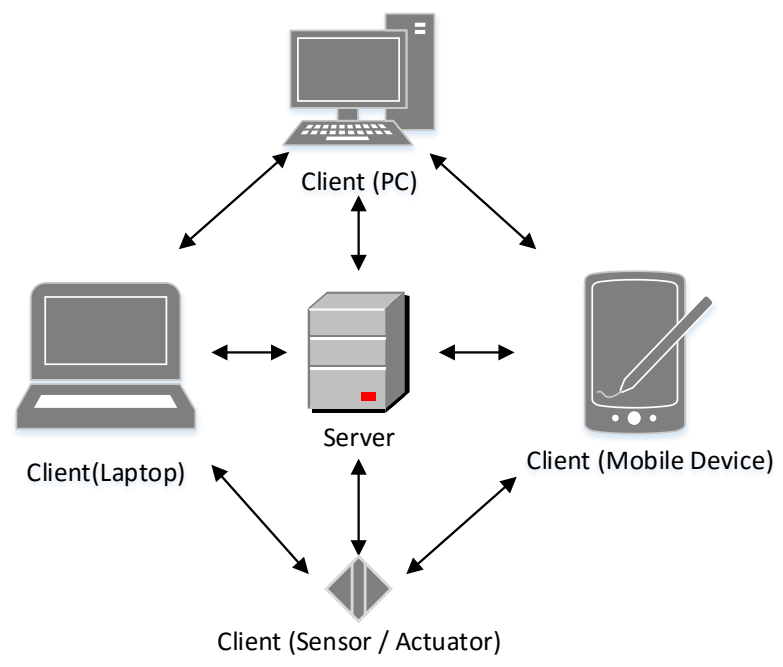

(b)

Fig. 2. (a) Client-server architecture, (b) Peer-to-peer client-server hybrid.

In all suggested topologies, sensors describe devices that detect player activity (for example location and movement), and transmit it either autonomously or through a sink node. Actuators detail interconnected devices that provide player feedback as a means of transcribing a game event such as level completion and confirmation of game event. Nodes comprise of actuators or sensors in all suggested topologies.

3) Decentralised Peer-to-Peer: Utilising a peer-to-peer topology for SSGs should consider the size and demand of the network required before being deployed. Dependant on the game requirements, a SSG could utilise this topology with no central server, but rather through computational devices that store and distribute game sections and interact with interconnected sensors and actuators based on a player's location, 


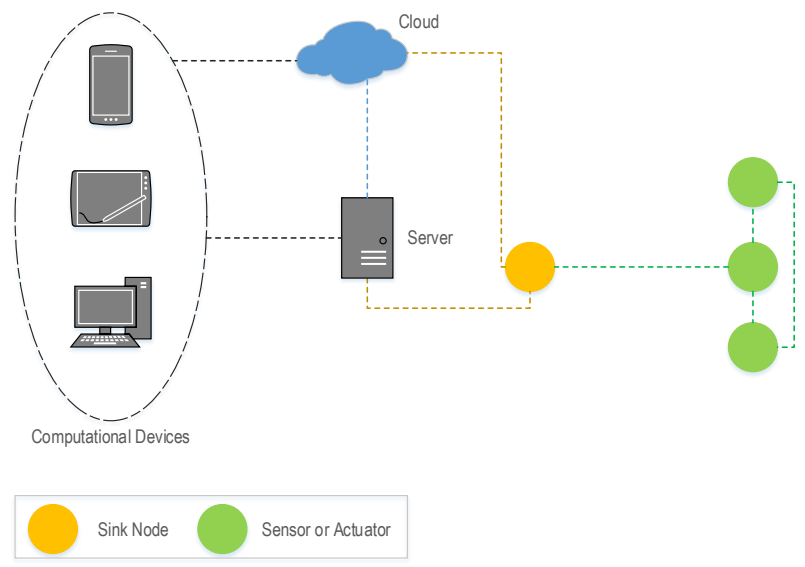

Fig. 3. Interconnected - Access Point Topology.

as illustrated in Fig. 4. Such a topology could be utilised for SSGs that are not resource demanding.

Although the processing power of smart devices is constantly increasing, they are still unable to store and process large virtual world data. Revisiting Father.io as an example, this game would suffer in an peer-to-peer topology due to the vast amount of location data that would need to be stored on smartphones and smartwatches. It could be argued that in the not so distant future, mobile smart devices may well be capable of doing so, however in the near future peer-to-peer topologies in SSGs should consider games with limited virtual environment.

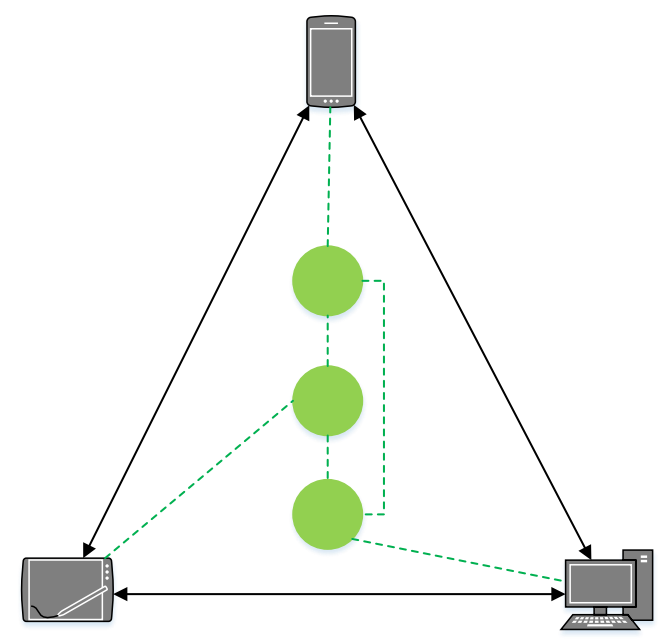

Fig. 4. Decentralised peer-to-peer Topology.

4) Hybrid: A hybrid WSN topology (Fig. 5) for SSGs would feature autonomous nodes, removing the sink node from the network architecture. By autonomous, this paper describes nodes with Internet connectivity. In this case the location of the nodes is dependant on the game requirements. Numerous locations can be used, providing that all positions provide access to Internet and power. Power management is a key element in hybrid topologies as the inclusion of connectivity requires more power for each node attached to the network. Hybrid topologies can be utilised for a variety of SSGs due to its versatility with deployment. As previously mentioned, Father.io could utilise a hybrid topology to provide all smart devices with autonomous connectivity. This improves the network robustness as it removes the dependency of a sink node.

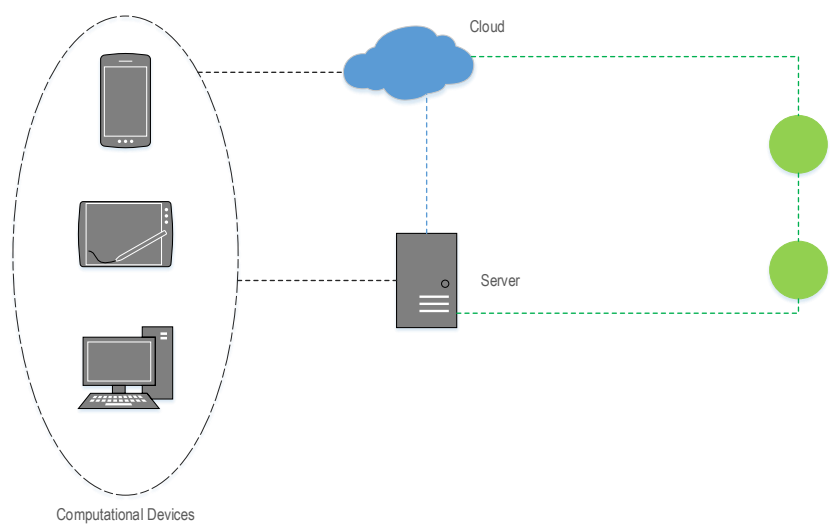

Fig. 5. Hybrid Topology.

It is important to adhere the requirements discussed including Scalability, QoS and Security, whilst considering the topology's robustness and reliability when utilising any of the aforementioned topologies.

A key difference between the use of topologies for SSGs and other uses such as WSN can be noted in the data that flows through the network. When considering a SSG topology, the data sourced from sensors and sent to actuators can be described as events. This is because any data that derives from a sensor or open source web datasets would inflict a change or an event in gameplay. For example, a Serious Game that monitors student engagement on a course would input a location check-in and translate it into a gameplay event such as user score, level up and increased inventory.

\section{APPLICATION OF SSG TOPOLOGIES}

This section discusses a proposed application for a SSG. This SSG aims to promote positive behavioural manipulation by increasing student engagement and illustrates its accompanied topology (Fig. 6). Games that utilise environmental and user data, sourced through smart devices and services to affect gameplay, could benefit from the proposition.

The SSG we propose to develop, to test the effectiveness of the topology, will utilise elements of question and answer to encourage players attention and focus. The content to be included for this game was influenced by the taxonomy published on Serious Games for Health issues. This taxonomy states for increasing attention and focus, question and answer games are preferred, and for behaviour modification, simulation and virtual games could be utilised [54]. Examples of questions that could be included into the SSG are: 


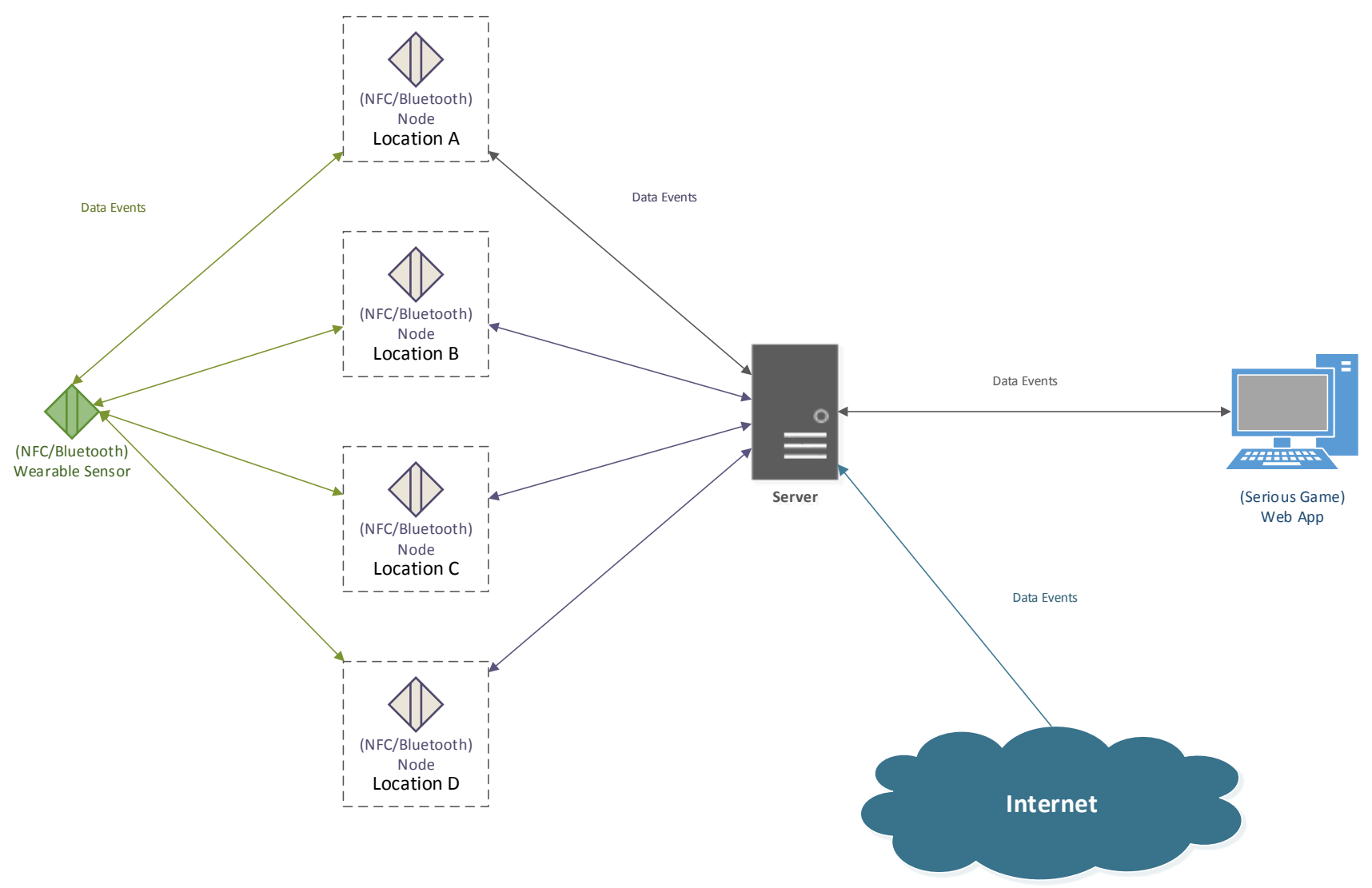

Fig. 6. Topology towards an SSG for behavioural modification.

1) Would you like to see how many points you need until the next level?

2) What do you believe is your monthly attendance percentage?

3) You did not attend yesterday's meeting. Would you like to reschedule one in your calendar?

Following, the elements of the topology are outlined and their purpose explained.

1) Wearable Sensor: WSN is chosen for this topology instead of Radio-Frequency Identification (RFID), to eliminate the need of a reader device [?] and create a more pervasive experience. It is important to aim for a pervasive solution as research has proven an increase in participant's perception for such solutions [39]. To track players location and attendance a wearable sensor will be provided with either Near-Field Communication (NFC) or Bluetooth capabilities. Further research will aim to justify the use of Sensors over RFID or NFC and validate the proposed topology, by cross-examining the two technologies.

2) Nodes: These nodes will utilise the campuses extended Wi-Fi coverage, relaying information to the central server, located on campus. In the SSG, registering check-ins into locations across the campus will be achieved through nodes with NFC or Bluetooth capabilities. Utilising players smartphones was considered to eliminate the need for static nodes, however due to battery life limitations on smartphones when utilising Bluetooth, we opted away from this solution. Cloud computing was considered for this project, but was not deemed vital in determining the effectiveness of SSGs' for behavioural modification.

3) Web Datasets: Datasets will be comprised of real world data in an attempt to correlate daily events with players behaviour. If such correlation can be achieved, the game will be able to better predict behaviour and encourage engagement in advance. An example of web datasets to be used include, sun cycle, weather and others. The number of datasets to be included greatly relies on availability of data.

4) Web Application: For behavioural modification instead of a game, based purely on simulation, we propose an avatar, 2D web application, which levels up and increases inventory based on users performance. As mentioned earlier, this decision derives from the Games for Health taxonomy [54].

5) Data Events: The data flowing through the illustrated topology is considered as data events. This is because all data generated and utilised (check-in, weather) will cause an immediate effect to the game. E.g. Reactive response: 
unlock inventory item for customisation of avatar, Proactive response: encourage players on a rainy day. Figure 4 notes a bi-directional relationship between all components of the suggested topology. By utilising bi-directional communication check-ins can be confirmed and the error percentage of data generated can be reduced. Errors can be reduced by ensuring data events have successfully been read and providing a form of feedback to players, such as haptic.

\section{RESEARCH CHALlENGES AND Future DiRECTIONS}

Developing a SSG, with today's technology advancements in mind, does present a number of challenges. Technical challenges involving scalability amass from the potential scale and diversity of networks. Games can involve sensors for input and gameplay alteration based on physical conditions. By utilising a network with large amounts of varied nodes generate limitations for service, data transfer, and networking [55].

Cost presents another technical challenge for developing SSGs. Though the price of sensors is decreasing, motion capture devices (such as Microsoft's Kinect) still remain somewhat expensive. Subscriptions to cloud storage and analysis companies and data management also addition to the total cost. The cost for a SSG solution currently varies greatly as it is dependant on the application.

Perhaps the most challenging technical issue for SSGs is the lack of heterogeneous resources. Manufacturers release their own applications and present data in numerous ways. Cloud services are attempting to tackle this issue, however further research and open source applications are required to bridge the gap [56]. This ties into the challenge of standardisation.

Standardisation promises a future in IoT where resources from a variety of services can interoperate on an application level. For SSGs this would allow input from unconventional data inputs to games today. In future, the data obtained from a car, a smart meter, online shopping habits could be cross-analysed and the player's carbon-footprint could be determined. After determining this, the game can suggest personalised methods for reducing the player's carbon footprint. Currently standardisation is an issue, with limited interoperability, limiting the potential for applications [55]. Further research is required to enhance standardisation. The standardisation of security policies and cyber-security in itself presents a further challenge.

Security presents challenges in different aspects including wireless networks, standards and cloud. WSNs are commonly utilised in IoT solutions, and SSGs are no different. The low energy requirements of WSNs require further research for more fitting security mechanisms [53]. Security policies require standardisation to allow multiple devices of various type to communicate securely and maintain data privacy [56]. Literature by D.Misha et.al. into cloud gaming uncovered the need for further research in cloud security for games before the technology can be widely adopted [46].

The aforementioned challenges only form a portion of the complete number of obstacles for developing a SSG in today's
IoT. Service specific issues will arise that have not been addressed in this section and will require further research.

\section{CONCLUSION}

To summarise, this paper presents the topologies that can be utilised for SSGs and a potential application of SSG, accompanied by the proposed topology.

After researching through the relative scientific fields and paradigms, three topologies were suggested; hybrid, interconnected access point and peer-to-peer. These topologies would cater for most games that utilise sensory input, distributed over a network.

The potential application of SSGs for manipulating behaviour, highlights the inclusion of real-world datasets for understanding the effects of everyday events on behaviour. A topology applied towards a SSG for behavioural manipulation is presented.

We accept that this research does not provide a complete illustration of all topologies and topological elements that can be utilised for developing SSGs and encourage further research to enhance this work. It is important to note, due to the new presence of SSGs in research, validation of the propositions is required through experimentation. The suggested application will validate the proposed topology in future works and findings will be published.

\section{REFERENCES}

[1] L. Atzori, A. Iera, and G. Morabito, "The Internet of Things: A survey," Computer Networks, vol. 54, no. 15, pp. 2787-2805, 2010.

[2] Z. Khalid, N. Fisal, and M. Rozaini, "A Survey of Middleware for Sensor and Network Virtualization," Sensors, vol. 14, no. 12, pp. 24046-24097, 2014.

[3] S. Tang, M. Hanneghan, and C. Carter, "A Platform Independent Game Technology Model for Model Driven Serious Games Development.," Electronic Journal of e-Learning, vol. 11, pp. 61-79, jan 2013.

[4] I. Blohm and J. M. Leimeister, "Gamification: Design of IT-based enhancing services for motivational support and behavioral change," Business and Information Systems Engineering, vol. 5, no. 4, pp. 275278, 2013.

[5] J. W. Burke, M. D. J. McNeill, D. K. Charles, P. J. Morrow, J. H. Crosbie, and S. M. McDonough, "Optimising engagement for stroke rehabilitation using serious games," Visual Computer, vol. 25, no. 12, pp. 1085-1099, 2009.

[6] C. Girard, J. Ecalle, and a. Magnan, "Serious games as new educational tools: How effective are they? A meta-analysis of recent studies," Journal of Computer Assisted Learning, vol. 29, no. 3, pp. 207-219, 2013.

[7] C. Aldrich, "The complete guide to simulations and serious games :how the most valuable content will be created in the age beyond Gutenberg to Google," Pfeiffer essential resources for training and HR professionals, vol. 1, p. 533, 2009.

[8] D. Sharma, J. C. Lakhmi, M. Favorskaya, and R. J. Howlett, Fusion of Smart, Multimedia and Computer Gaming Technologies. Springer International Publishing, 1 ed., 2015.

[9] E. Lotfi and B. Mohammed, "Players Performances Analysis based on Educational Data Mining Case of Study : Interactive Waste Sorting Serious Game," International Journal of Computer Applications, vol. 108, no. 11, pp. 13-18, 2014.

[10] L. Elaachak, A. Belahbibe, and M. Bouhorma, "Towards a System of Guidance, Assistance and Learning Analytics Based on Multi Agent System Applied on Serious Games," International Journal of Electrical and Computer Engineering (IJECE), vol. 5, no. 2, pp. 344-354, 2015.

[11] G. Armitage, M. Claypool, and P. Branch, Networking and Online Games. Wiley-Blackwell, 2006.

[12] K. Ashton, "That 'Internet of Things' Thing," RFID Journal, vol. 22, jun 2009. 
[13] Efficient particle filter localization algorithm in dense passive RFID tag environment, vol. 61. IEEE Transactions on Industry Electronics, Vol 61, Issue 10, 2014.

[14] E. Welbourne, L. Battle, G. Cole, K. Gould, K. Rector, S. Raymer, and M. Balazinska, "Building the Internet of Things Using RFID," Internet Computing, IEEE, vol. 13, no. 3, pp. 48 - 55, 2009.

[15] P. Yang, "Improving Passive RFID Localisation Precision with Moving Direction Estimation based FeatureImprovement," IET Wireless Sensor Systems Journal, vol. 4, no. 1, 2014.

[16] Y. Po, W. Wenyan, M. Moniri, and C. C. Chibelushi, Efficient Object Localization Using Sparsely Distributed Passive RFID Tags, vol. 60. IEEE Transactions on Industry Electronics, Vol 60, Issue 12, 2013.

[17] P. Yang, Experimental Evaluation and Analysis of Achieving High Accuracy and Precision in Passive HF RFID Location Systems, vol. 2. 2015.

[18] X. Jia, Q. Feng, T. Fan, and Q. Lei, "RFID technology and its applications in Internet of Things (IoT)," 2012 2nd International Conference on Consumer Electronics, Communications and Networks (CECNet), vol. 21, pp. 1282-1285, 2012.

[19] C. Sun, "Application of RFID technology for logistics on internet of things," AASRI Procedia, vol. 1, pp. 106-111, 2012.

[20] P. C., Z. A., C. P., and G. D., "Sensing as a service model for smart cities supported by Internet of Things," European Transactions on Telecommunications, vol. 25, pp. 81-93, jan 2014.

[21] A. Zanella, N. Bui, A. Castellani, L. Vangelista, and M. Zorzi, "Internet of things for smart cities," Internet of Things Journal, IEEE, vol. 1, pp. 22-32, feb 2014.

[22] J. Guerrero Ibanez, S. Zeadally, and J. Contreras-Castillo, "Integration challenges of intelligent transportation systems with connected vehicle, cloud computing, and internet of things technologies," IEEE Wireless Communications, vol. 22, no. 6, pp. 122-128, 2015.

[23] Z. Deng, P. Yang, Y. Zhao, X. Zhao, and F. Dong, Life-logging data aggregation solution for interdisciplinary healthcare research and collaboration. 2015.

[24] P. Yang, M. Hanneghan, J. Qi, Z. Deng, D. Fan, and F. Dong, Improving the Validity of Lifelogging Physical Activity Measures in an Internet of Things Environment. 2015.

[25] E. G. Spanakis, D. Kafetzopoulos, P. Yang, K. Marias, Z. Deng, M. Tsiknakis, V. Sakkalis, and F. Dong, MyHealthAvatar: Personalised and empowerment health services through Internet of Things technologies. 2014.

[26] Y. Wu, Q. Z. Sheng, and S. Zeadally, RFID: Opportunities and challeges, pp. 105-129. London: Springer London, 2013.

[27] D. Guinard, S. Karnouskos, V. Trifa, B. Dober, P. Spiess, and D. Savio, "Interacting with the SOA - based Internet of Things : Discovery, Query , Selection , and On - Demand Provisioning of Web Services," IEEE Transactions on Services Computing, vol. 3, no. 3, pp. 223-235, 2010.

[28] B. Y. Xu, L. D. Xu, and E. H. M. Cai, Ubiquitous data accessing method in IoT-based information system for emergency medical services, vol. 10. Issue: 2, 2014.

[29] S. Fang, L. Xu, H. Pei, Y. Liu, Z. Liu, Y. Zhu, J. Yan, and H. Zhang, “An Integrated Approach to Snowmelt Flood Forecasting in Water Resource Management," Industrial Informatics, IEEE Transactions on, vol. 10, pp. $548-558$, feb 2013.

[30] J. Gubbi, R. Buyya, S. Marusic, and M. Palaniswami, "Internet of Things (IoT): A vision, architectural elements, and future directions," Future Generation Computer Systems, vol. 29, no. 7, pp. 1645-1660, 2013.

[31] E. Fleisch, "What is the Internet of Things? When things add value," White Paper, vol. 8, 2010.

[32] J. Gregory, Game Engine Architecture, Second Edition. CRC Press, second ed., 2014.

[33] S. Tang and M. Hanneghan, "State-of-the-Art Model Driven Game Development: A Survey of Technological Solutions for Game-Based Learning," Journal of Interactive Learning Research, vol. 22, no. 4, pp. 551-605, 2011.

[34] B. Sawyer and P. Smith, "Serious games taxonomy," Slides from the Serious Games Summit at the Game ..., pp. 1-54, 2008.

[35] C. Hunter, "Modeling, Molecular Machinery: Activities, Simulations, and Computer-Modeling Programs That Help Students Visualize How Proteins Work," The Science Teacher, vol. 82, no. 2, p. 49, 2015.

[36] J. L. Anderson and M. Barnett, "Learning Physics with Digital Game Simulations in Middle School Science," Journal of Science Education and Technology, vol. 22, no. 6, pp. 914-926, 2013.
[37] J. Difede, B. O. Rothbaum, G. Reger, J. Spitalnick, J. Cukor, R. Mclay, and A. Rizzo, "Development and early evaluation of the Virtual Iraq/Afghanistan exposure therapy system for combat-related PTSD," Ann. N.Y. Acad. Sci.

[38] T. H. Laine and C. I. Sedano, "Distributed Pervasive Worlds : The Case of Exergames," Journal of Educational Technology \& Society, vol. 18, no. 1, pp. 50-66, 2015.

[39] Z. Lv, A. Halawani, S. Feng, S. ur Réhman, and H. Li, "Touch-less interactive augmented reality game on vision-based wearable device," Personal and Ubiquitous Computing, pp. 551-567, 2015.

[40] M. Patel and J. Wang, "Applications, challenges, and prospective in emerging body area networking technologies," IEEE Wireless Cоттиnications, vol. 17 , no. 1 , pp. 80-88, 2010.

[41] I. Blohm and J. M. Leimeister, "Gamification: Design of IT-based enhancing services for motivational support and behavioral change," Business and Information Systems Engineering, vol. 5, no. 4, pp. 275278, 2013.

[42] R. Adelmann, M. Langheinrich, and C. Flörkemeier, "Toolkit for bar code recognition and resolving on camera phones-jump starting the internet of things.," GI Jahrestagung (2), vol. 94, pp. 366-373, 2006.

[43] T. H. Laine and C. I. Sedano, "Distributed Pervasive Worlds : The Case of Exergames," Journal of Educational Technology \& Society, vol. 18, no. 1, pp. 50-66, 2015.

[44] A. Yahyavi and B. Kemme, "Peer-to-peer architectures for massively multiplayer online games," ACM Computing Surveys, vol. 46, no. 1, pp. 1-51, 2013.

[45] A. P. Yu and S. T. Vuong, "MOPAR : A Mobile Peer-to-Peer Overlay Architecture for Interest Management of Massively Multiplayer Online Games," Acm, pp. 99-104, 2005.

[46] D. Mishra, M. El Zarki, A. Erbad, C. H. Hsu, N. Venkatasubramanian, and M. E. Zarki, "Clouds + Games: A multifaceted approach," IEEE Internet Computing, vol. 18, pp. 20-27, 2014.

[47] C. Alcaraz, P. Najera, J. Lopez, and R. Roman, "Wireless Sensor Networks and the Internet of Things : Do We Need a Complete Integration ?", 1st International Workshop on the Security of the Internet of Things (SecIoT'10), no. October 2015, pp. 1-8, 2010.

[48] D.-g. Zhang, Y.-n. Zhu, C.-p. Zhao, and W.-b. Dai, "A new constructing approach for a weighted topology of wireless sensor networks based on local-world theory for the Internet of Things (IOT)," Computers \& Mathematics with Applications, vol. 64, pp. 1044-1055, sep 2012.

[49] S. F. Ochoa and R. Santos, "Human-centric wireless sensor networks to improve information availability during urban search and rescue activities," Information Fusion, vol. 22, pp. 71-84, 2013.

[50] I. Akyildiz, W. Su, Y. Sankarasubramaniam, and E. Cayirci, "Wireless sensor networks: a survey," Computer Networks, vol. 38, no. 4, pp. 393422, 2002.

[51] G. Schiele, R. Suselbeck, A. Wacker, J. Hahner, C. Becker, and T. Weis, "Requirements of Peer-to-Peer-based Massively Multiplayer Online Gaming," Seventh IEEE International Symposium on Cluster Computing and the Grid CCGrid 07, pp. 773-782, 2007.

[52] M. Zorzi, A. Gluhak, S. Lange, and A. Bassi, "From today's INTRAnet of things to a future INTERnet of things: A wireless- and mobilityrelated view," IEEE Wireless Communications, vol. 17, no. 6, pp. 44-51, 2010.

[53] D. Christin, A. Reinhardt, P. S. Mogre, and R. Steinmetz, "Wireless Sensor Networks and the Internet of Things : Selected Challenges," Proceedings of the 8th GI/ITG KuVS Fachgespräch Drahtlose Sensornetze, pp. 31-34, 2009.

[54] D. H. Goh, R. P. Ang, and H. C. Tan, "Strategies for designing effective psychotherapeutic gaming interventions for children and adolescents," Computers in Human Behavior, vol. 24, no. 5, pp. 2217-2235, 2008.

[55] D. Miorandi, S. Sicari, F. De Pellegrini, and I. Chlamtac, "Internet of things: Vision, applications and research challenges," 2012.

[56] L. D. Xu, W. He, and S. Li, "Internet of things in industries: A survey," IEEE Transactions on Industrial Informatics, vol. 10, no. 4, pp. 22332243,2014 